\title{
Aging studies of the triple-GEM detectors for future upgrades of the CMS muon high rate region at the HL-LHC
}

\author{
Yong Hoon Lee ${ }^{* a}$ and Francesco Fallavollita ${ }^{b}$ on behalf of the CMS Muon Group \\ ${ }^{a}$ Sungkyunkwan University \\ Suwon, Korea \\ ${ }^{b}$ Pavia University and INFN Pavia \\ Pavia, Italy \\ E-mail: yong.hoon.lee@cern.ch, francesco.fallavollita@cern.ch
}

\begin{abstract}
The high-luminosity LHC (HL-LHC) upgrade is setting a new challenge for particle detection technologies. In the CMS muon system based on gas detectors, the increased luminosity will yield a ten times higher particle background compared to the present LHC conditions. To cope with the high-rate environment and to maintain the actual performance, new Gas Electron Multiplier (GEM) detectors will be installed in the innermost region of the forward CMS muon spectrometer, $2<\eta<2.8$ (ME0 project). The detailed knowledge of the detector performance in the presence of such a high background is crucial for an optimized design and efficient operation at the HLLHC. A precise understanding of possible aging effects of detector materials and gases is of extreme importance. For this reason, aging tests of full sized triple-GEM detector operated with an $\mathrm{Ar} / \mathrm{CO}_{2}$ (70/30) gas mixture at an effective gas gain of $2 \times 10^{4}$, are in course at GIF++, the CERN Gamma Irradiation Facility. One detector is irradiated with $662 \mathrm{keV}$ gamma - rays from a $14 \mathrm{TBq}{ }^{137} \mathrm{Cs}$ source and, in parallel, a second similar detector with $22 \mathrm{keV} \mathrm{X}$-rays at the quality control lab. This contribution describes the performance of triple-GEM detectors during the irradiation test and reports on their state-of-the art.
\end{abstract}

The 39th International Conference on High Energy Physics (ICHEP2018)

4-11 July, 2018

Seoul, Korea

${ }^{*}$ Speaker. 


\section{Introduction}

The goal of the CMS GEM aging test campaign at the CERN Gamma Irradiation Facility (GIF++) [1] and in the CMS-GEM Production Lab. [2] is to qualify ten years of CMS-GEM operation at HL-LHC, with a minimum safety factor of three.

\section{Aging studies of CMS triple-GEM detectors}

The result for the anode current shown in Figure 1(a) indicates that the CMS triple-GEM detector does not suffer from any kind of aging effects or long-term degradation on GIF++[3].

An aging test with high rate $22 \mathrm{keV} \mathrm{X}$ - rays source was also set up in summer 2017 on CMSGEM Production Lab. Not only monitor the anode current, also measure effective gain and energy resolution every week, i.e. every $30 \mathrm{mC} / \mathrm{cm}^{2}$ of accumulated charge (Figure 1(c)).

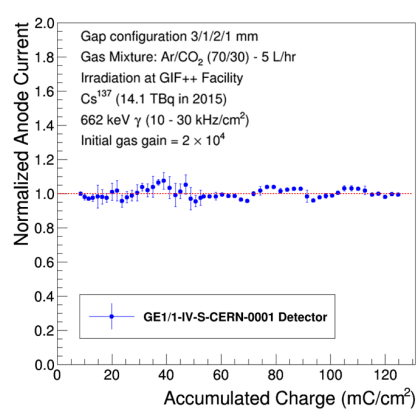

(a)

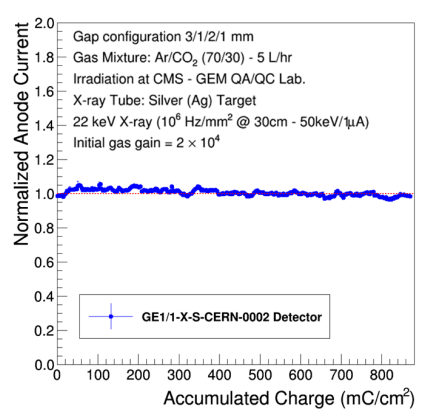

(b)

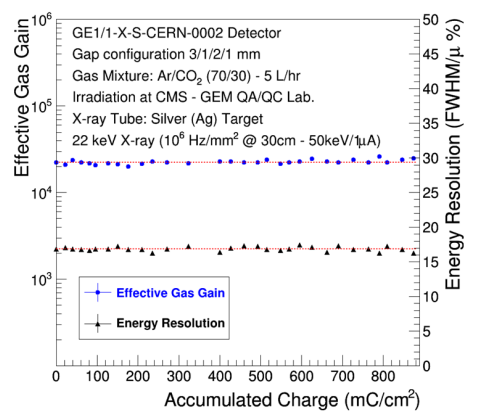

(c)

Figure 1: Figure 1(a): Result of the GEM aging test at GIF++ showing the normalized and corrected anode current as a function of the accumulated charge. Figure 1(b): Result of the GEM aging test with $\mathrm{X}$ - ray showing the normalized and corrected anode current as a function of the accumulated charge. Figure 1(c): Result of the GEM aging test with $\mathrm{X}$ - ray showing the normalized corrected gain and energy resolution as a function of the accumulated charge.

\section{Conclusion}

The preliminary results presented in this paper indicate that the CMS triple-GEM detector can sustain the continuous operation in the CMS endcap environment for over 10 years at HL-LHC without suffering from any performance degradation.[2].

\section{References}

[1] M. R. Jäkel et al., CERN GIF++:A new irradiation facility to test large-area particle detectors for the high-luminosity LHC program, in proceedings of 3rd International Conference on Technology and Instrumentation in Particle Physics, TIPP 2014.

[2] A. Colaleo et al., CERN-LHCC-2017-012 CMS-TDR-016.

[3] J. A. Merlin, Study of long-term sustained operation of gaseous detectors for the high rate rnvironment in CMS, PhD Thesis, CERN-THESIS-2016-041. 